\title{
Rain attenuation statistics over 5G millimetre wave links in malaysia
}

\author{
Mustafa Ghanim', Manhal Alhilali ${ }^{2}$, Jafri Din ${ }^{3}$, Hong Yin Lam ${ }^{4}$ \\ 1,2,3Wireless Communication Centre, Faculty of Electrical Engineering, Universiti Teknologi Malaysia, Malaysia \\ ${ }^{4}$ Faculty of Engineering Technology, Universiti Tun Hussein Onn Malaysia, Hab Pendidikan Tinggi Pagoh, Malaysia
}

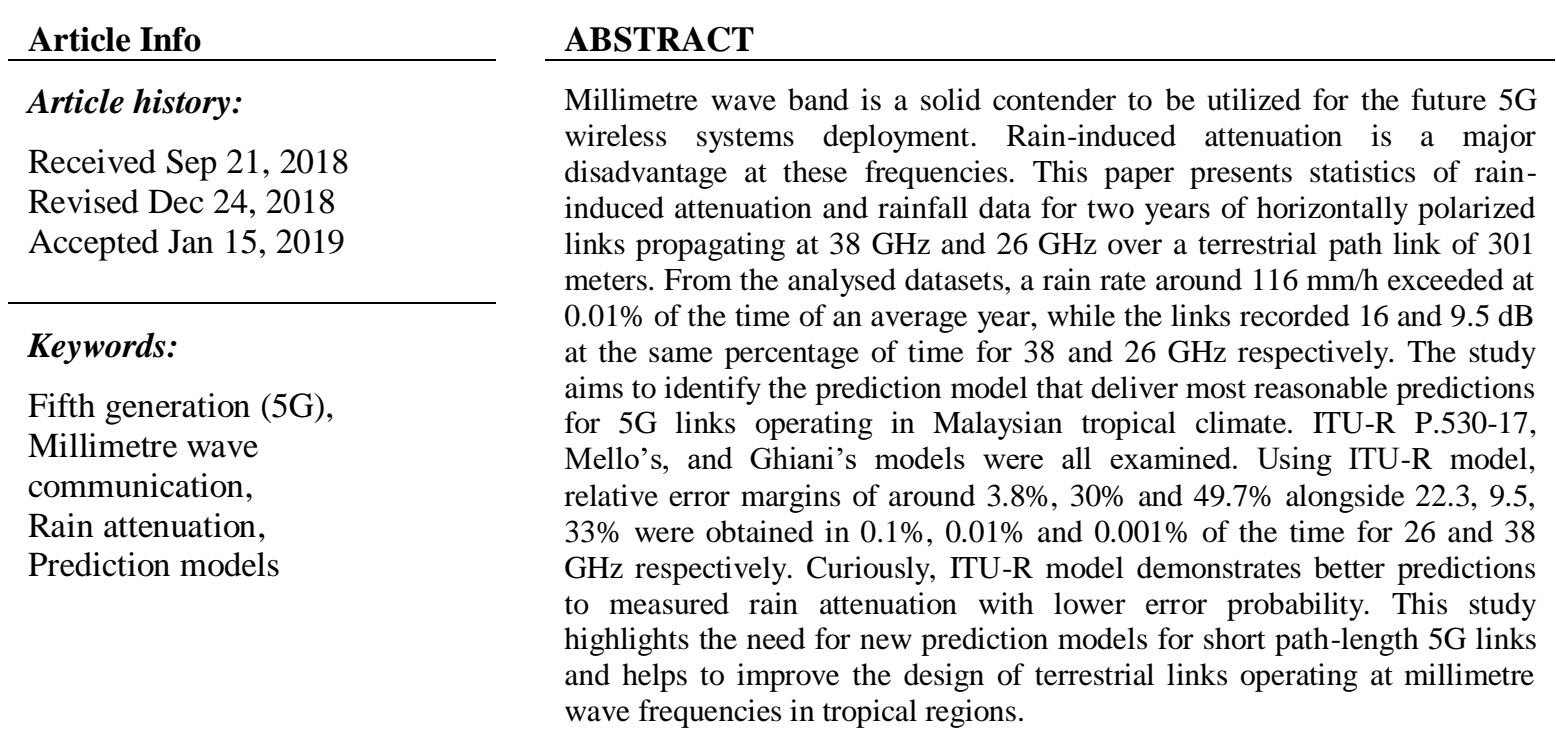

Copyright $\odot 2019$ Institute of Advanced Engineering and Science. All rights reserved.

\section{Corresponding Author:}

Jafri Din,

Wireless Communication Centre, Faculty of Electrical Engineering,

Universiti Teknologi Malaysia,

81310 Johor Bahru, Johor, Malaysia.

Email: jafri@utm.my

\section{INTRODUCTION}

For the currently deployed 4th generation of wireless communications, excess attenuation due to rain can be neglected. However, the demand for greater bandwidth by telecommunication providers requires the move to higher frequency bands in the implementation of the next 5th generation (5G) wireless communication [1]. In tropical regions, the occurrence of rain is more frequent, and the effect on radio links becomes dramatically high for frequencies above $7 \mathrm{GHz}$, due to higher rain intensities [2], [3]. Therefore, it is fundamental to precisely predict rain-induced attenuation for the planning and design of higher capacity point-to-point 5G radio communication system [4]. The 1-minute rain rate statistics and the rain rate exceeded at a certain percentage of the average year are the most broadly utilized parameters to determine the rain-induced attenuation [5], [6]. Numerous models have been created utilizing these parameters to predict the rain-induced attenuation. However, the majority of these models are conducted and focused on the temperate regions [7]. This could create an issue when applied to the tropical countries due to higher rain rates in tropical regions in comparison to temperate regions. To that end, A campaign to measure the rain attenuation statistics was carried in Universiti Teknologi Malaysia (UTM) [8]. Some of the most widely used prediction models are empirically based, which uses historical databases for given geographical regions [9], while others are physically based, designed to recover the physical attenuation process, which in turns 
requires more parameters and longer time to process [10]. Many studies have analysed rain attenuation extensively and various models to overcome the issue have been developed [9]-[12] that primarily enhance the ITU-R P. 530-17 [13] for specific scenarios.

This study investigate three established rain attenuation models, selected to address different types and assumptions used while designing the models, namely, ITU-R P. 530-17 [13] which is the most widely used, Mello model [9] which is usually used in tropical regions, Ghiani's model [14] which is a new physical based model.

The following sections are organized as follow: In Section 2, prediction models used for the estimation of rain attenuation are briefly discussed. Followed by the system setup, and data collection procedure is discussed. Result and discussion section summarize the accuracy of prediction models. Finally, a conclusion is given in Section 5, and the possible future research based on the findings is presented.

\section{RAIN ATTENUATION PREDICTION MODELS}

Rain-induced attenuation usually presented as the product of rain specific attenuation $\gamma_{R}(\mathrm{~dB} / \mathrm{km})$ and the effective propagation path-length $d_{\text {eff }}(\mathrm{km})$ [15]. The rain-induced attenuation, $A(\mathrm{~dB})$, above $p$ percent of time is then defined as:

$$
A=\gamma_{R} d_{e f f}=\gamma_{R} d * r
$$

where $d$ is the actual path-length in $\mathrm{km}$, and $r$ is path reduction factor at $p$ percentage of time.

In the ITU-R recommendation P.838-3 [15], the technique to calculate the specific attenuation from the rain intensity was described. The specific rain attenuation, $\gamma_{R}(\mathrm{~dB} / \mathrm{km})$ is computed from the rain rate $R$ $(\mathrm{mm} / \mathrm{h})$ exceeded at $p$ percent of the time by applying the power-law relationship as,

$$
\gamma_{R}=k R^{\alpha}
$$

where, $k$ and $\alpha$ are frequency dependent coefficients. These constants can be found in the recommendation tables. Due to the rainfall non-uniformity along the propagation path, a reduction factor of the actual link path-length is presented to reduce the effective path length [10]. However, for shorter links the reduction factor takes values larger than unity, to overcome the model's initial design assumptions. Each model addresses this problem in different way. In this paper three different models included, those of the ITU-R Model [13], Mello's model [9], Ghiani's model [14], these models are briefly explained in the following subsections.

\subsection{ITU-R P.530.17 Model}

The ITU-R recommendation [13] presents the rain attenuation, as described earlier in 2, depending on the path reduction factor, which considers the time-space variability of rain intensity along path. The following reduction factor is presented

$$
r=\frac{1}{0.477 d^{0.633} R_{0.01}^{0.073 \propto} f^{0.123}-10.579(1-\exp (-0.024 d))}
$$

where $r$ is the distance factor, $f$, is the frequency in $\mathrm{GHz}, d$, is the distance. The maximum value of $r$ is capped at 2.5 .

The resulted rainfall attenuation value exceeded at $0.01 \%$ of the time in an average year, is scaled by an empirical formula to other percentages of time between $1 \%$ and $0.001 \%$ by using the following equation:

$$
\left.\frac{A_{p}}{A_{0.01}}=C_{1} p^{(} C_{2}+C_{3} \log 10 p\right)
$$

where,

$$
\left\{\begin{array}{c}
C_{1}=\left(0.07^{c_{0}}\right)\left(0.12^{\left(1-c_{0}\right)}\right) \\
C_{2}=0.855 C_{0}+0.546\left(1-C_{0}\right) \\
C_{3}=0.139 C_{0}+0.043\left(1-C_{0}\right)
\end{array}\right.
$$

And, 


$$
C_{0}=\left\{\begin{array}{c}
0.12+0.4\left(\log _{10}\left(\frac{f}{10}\right)^{0.8} f \geq 10 G H z\right. \\
0.12 f<10 G H z
\end{array}\right\}
$$

This model is recommended to be used worldwide, by providing locally measured rain rates, or by using ITU-R maps, it can help in the prediction for any frequency between 1 and $100 \mathrm{GHz}$ with path-lengths up to $60 \mathrm{~km}$.

\subsection{Mello's Model}

This model uses multiple non-linear regressions to obtain the required numerical coefficients from rain rate and rain cell diameter. And was initially designed using the databanks provided the ITU-R. Mello [9] have used similar procedure as done in [13] which is used by the presented ITU-R model, and overcome the limitation of the ITU-R model by using the complete rainfall distribution as the models input, and keep using the reduction factor parameters from the ITU-R model. In order to correct these limitations, a new effective rainfall rate coefficient $\left(R_{e f f}\right)$ was developed., which helps to predict the rain-induced attenuation complementary cumulative distribution function (CCDF) instead of a single percentage of time, and can be computed as:

$$
A_{p}=\gamma_{R} \cdot d_{e f f}=k\left(R_{e f f}(R, d)\right)^{\alpha} \frac{1}{1+\frac{d}{d_{0}(R)}}
$$

where,

$$
d_{e f f}=\frac{1}{1+\frac{d}{d_{0}}} d=r d
$$

here $d$ is the link path-length, $r$ as presented earlier, the path reduction factor. The definition for $R_{e f f}$ and equivalent rain cell diameter $d_{0}$ is then given by:

$$
\begin{aligned}
& R_{e f f}=1.763 R^{0.753+\frac{0.197}{d}} \\
& d_{0}=119 R^{-0.244}
\end{aligned}
$$

Multiple nonlinear regressions are used to obtain the numerical coefficients in (9) and (10), using the databanks provided the ITU-R.

\subsection{Ghiani Model}

Ghiani [14] has developed novel physically based approach to model the rain attenuation affecting terrestrial links. This model is devised by simulating the interaction between terrestrial links and synthetic rain maps, which are specifically exploited to investigate the path reduction factor, considering the spatial inhomogeneity of rainfall along the link. The accuracy of the proposed model was tested against the MultiEXCELL-derived rain attenuation statistics and against the independent set of experimental data included in the DBSG3 database made available by ITU-R. The rain attenuation exceeded with probability $p$ in an average year can be calculated as

$$
A(p, d)=K R(p)^{\propto} d\left[a(d) e^{-b(d) R(p)}+c(d)\right]
$$

where $R(p)$, extracted from the local input rain rate complementary cumulative distribution function $(\mathrm{CCDF})$, is the rain rate exceeded with probability $p$, coefficients $a, b$, and $c$ are defined as:

$$
\left\{\begin{array}{c}
\mathrm{a}=-0.8743 e^{-0.1111 d}+0.9061 \\
\mathrm{~b}=-0.0931 e^{-0.0183 d}+0.1002 \\
\mathrm{c}=-0.6613 e^{-0.178 d}+0.3965
\end{array}\right.
$$

\section{SYSTEM SETUP AND DATA COLLECTION}

Two experimental millimetre wave links operates at 26 and $38 \mathrm{GHz}$ were mounted at UTM Johor campus [5]. The links were set between the roof of microwave lab and the second transceiver was installed 
on a telecom tower with a path-length between the two antennas of 301 meters. Both antennas are covered by radomes to prevent wetting antenna conditions. The Automatic gain control output level of the antenna unit is then connected to a PC using a data acquisition system and the sampling interval was set to 1-second. The logged data then stored into databank using $\mathrm{C}$ language written. The data was collected over duration of 24 months and a satisfactory data availability of $99.95 \%$ was achieved.

A tipping-bucket rain gauge with tip size of $0.2 \mathrm{~mm}$ was set up on the same roof. The rain gauge added a time stamp for each tip with 0.1 second resolution. Similar method to calculate 1 -minute rain rate used in [16] is used for to present the data in 1-minute integration time. For $0.01 \%$ of the time the 1-minute rain rate was found to be around $116 \mathrm{~mm} / \mathrm{h}$ at the measurement site. Figure 1 show the rain-induced attenuation versus rain rate for both investigated links of 38 and $26 \mathrm{GHz}$. The attenuation reaches up to $25 \mathrm{~dB}$ on the $38 \mathrm{GHz}$ link, and 16 on the $26 \mathrm{GHz}$ link. This highlight that even with short link distance, that will be used for $5 \mathrm{G}$ applications, the rain attenuation should not be underestimated.

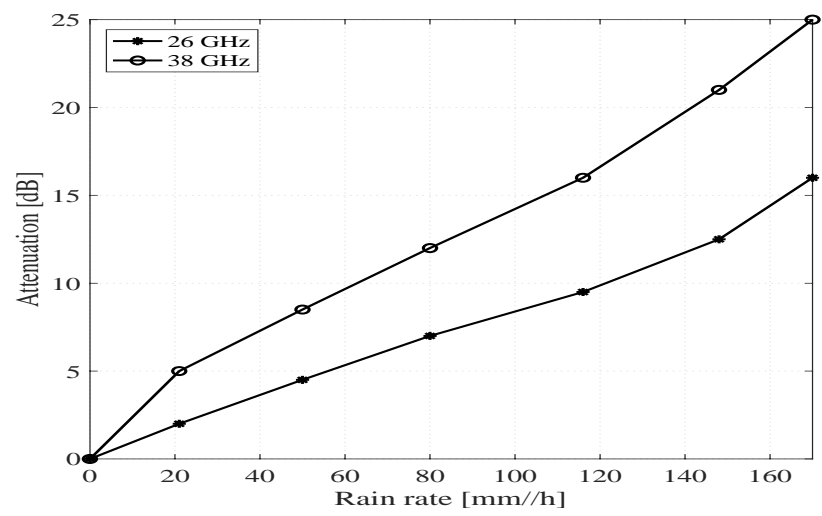

Figure 1. Distribution of rain attenuation versus rain rate

\section{RESULT AND DISCUSSIONS}

Figure 2 presents the prediction error plots of ITU-R Model versus rainfall rates for 38 and $26 \mathrm{GHz}$ under horizontal polarization respectively.

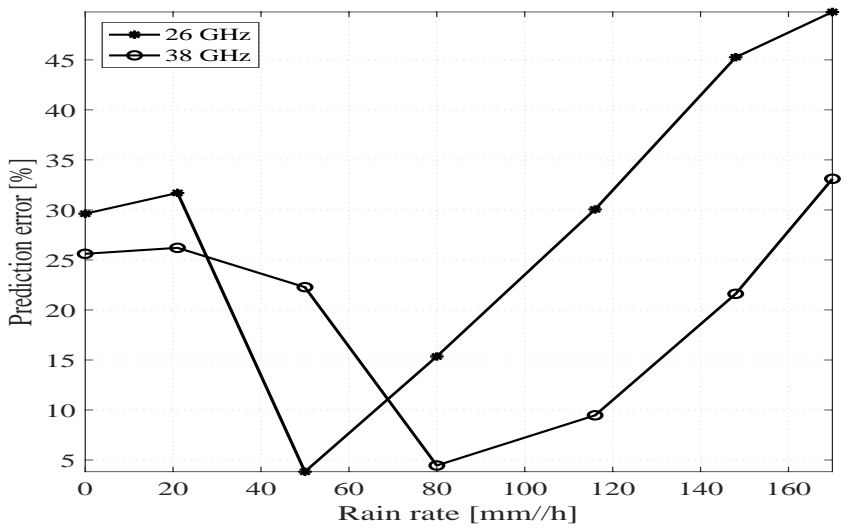

Figure 2. ITU-R P.530-17 prediction error with rain rate

It was found that the error in prediction is higher for $38 \mathrm{GHz}$ link as compared to $26 \mathrm{GHz}$. The prediction error increases with higher rain rate, due to the initial design of the model based on the data bank from temperate regions, where the rain rates will not reach these high values.

Figure 3 present comparison between the measured values for 38 and $26 \mathrm{GHz}$ links, and the selected attenuation models. The ITU-R prediction slightly overestimated the rain attenuation and the highest disparity was $8 \mathrm{~dB}$ at $38 \mathrm{GHz}$ link. The disparity might be due to the fact that the initial design factors investigated to predict the rain attenuation in the model have not considered the propagation tropical rain. 
Additionally, ITU-R P. 530-17 is designed for link with path-length longer than $1 \mathrm{~km}$. ITU-R P.530-17, and Ghiani's models give closer estimates to the measured CCDFs of rain attenuation. Large overestimate is shown by Mello's model. The ITU-R model prediction was the most suitable and generated fewer disparities between measured rain attenuation values and the predictions in comparison to other models.

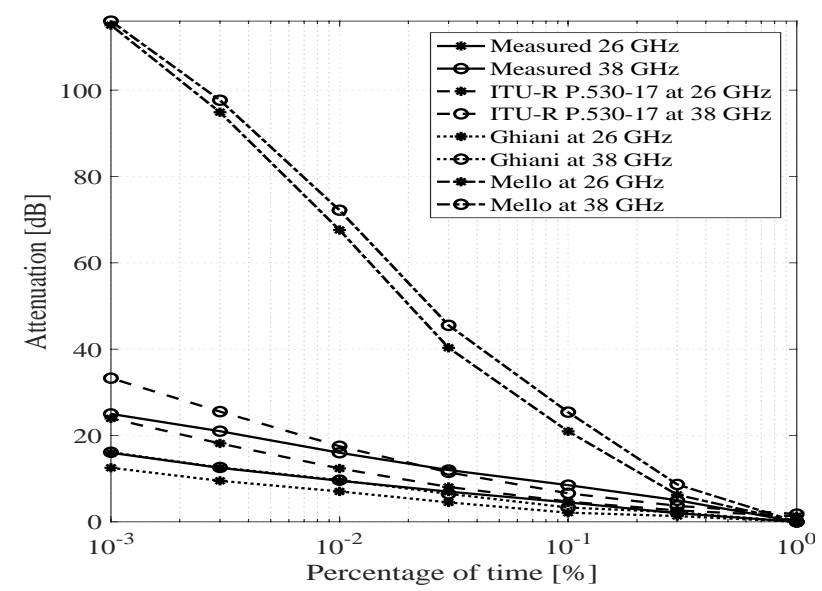

Figure 3. CCDF of rain attenuation compared with prediction models at various time percentages for 38 and $26 \mathrm{GHz}$

The Mello model shows excessive overestimation. As an example, Mello predicts 25.4, 72.2, 116 $\mathrm{dB}$ at $0.1 \%, 0.01 \%, 0.001 \%$ of the time for $38 \mathrm{GHz}$ link, this might be due to the design of the model based on long-path links. Table 1 shows the measured and predicted attenuation values for 26 and $38 \mathrm{GHz}$ links at critical percentages of the time.

Table 1. Attenuation Values for $0.1,0.01,0.001 \%$ of the Time for 26 and 38 Ghz Links

\begin{tabular}{ccccccccc}
\hline \multicolumn{1}{c}{$26 \mathrm{GHz}$} \\
\hline \multirow{2}{*}{ Time percentage $(\%)$} & $\begin{array}{c}\text { measured } \\
(\mathrm{dB})\end{array}$ & $\begin{array}{c}\text { ITU-R } \\
(\mathrm{dB})\end{array}$ & Ghiani & Mello & measured & ITU-R & Ghiani & Mello \\
$(\mathrm{dB})$ & $(\mathrm{dB})$ & $(\mathrm{dB})$ & $(\mathrm{dB})$ & $(\mathrm{dB})$ & $(\mathrm{dB})$ \\
0.1 & 4.5 & 4.67 & 2.12 & 20.96 & 8.5 & 6.6 & 3.31 & 25.4 \\
0.01 & 9.5 & 12.35 & 7.04 & 67.61 & 16 & 17.51 & 9.65 \\
0.001 & 16 & 23.97 & 12.52 & 115.1 & 25 & 33.27 & 16.11 & 72.2 \\
\hline
\end{tabular}

The relative error figure $(\varepsilon(p))$, is presented to measure the appropriateness for each of the model. And further comparisons are done through the calculation of Standard Deviation (STD) and Root Mean Square (RMS), to examine the performance of the ITU-R, Mello's, and Ghiani's models. The error figure used to examine the accuracy of the prediction models is given by:

$$
\varepsilon(p)=\frac{A_{p, \text { predected }}-A_{p, \text { measured }}}{A_{p, \text { measured }}} \times 100
$$

The calculated $\varepsilon(p)$, Standard Deviation (STD) and Root Mean Square (RMS) means for each model are listed in Table 2 for 26 and $38 \mathrm{GHz}$ respectively.

As can be noted from the table, Mello's model shows higher relative error compared to ITU-R model and Ghiani's models, which is apparent with the increased STD and RMS values. For that, ITU-R, Ghaini's models give better prediction alongside the measured rain attenuation.

Table 2. Percentage Error Obtained Over the Interval $0.001-1 \%$ for $26,38 \mathrm{Ghz}$

\begin{tabular}{ccccccc}
\hline & \multicolumn{3}{c}{$26 \mathrm{GHz}$} & \multicolumn{3}{c}{$38 \mathrm{Ghz}$} \\
\hline & ITU-R & Ghiani & Mello & ITU-R & Ghiani & Mello \\
\hline $\boldsymbol{\varepsilon}(\boldsymbol{p}) \boldsymbol{m e a n}$ & 29.3 & 32.2 & 406.43 & 1.8 & 46.4 & 218.8 \\
\hline RMS & 32.8 & 33.9 & 479.26 & 21.8 & 47.3 & 273.1 \\
\hline STD & 16.4 & 11.4 & 174.5 & 23.8 & 10.1 & 116.9 \\
\hline
\end{tabular}




\section{CONCLUSION}

Based on the presented results for the described experiment on 26 and $38 \mathrm{GHz}$ links, the rain rate and the induced attenuation are presented for $5 \mathrm{G}$ short path-length links. Three well established rain attenuation models were used to compare the predicted results with the measured data. The presented comparison shows that ITU-R P.530-17 model was the most appropriate to be used under the link specification and tropical climate, by giving closer predictions to the measured rain induced attenuation. However, for the lower frequency $26 \mathrm{GHz}$, the prediction error was slightly lower in comparison with 38 $\mathrm{GHz}$, this might be due to the differences in rain drop size distribution effects on higher frequencies. Additionally, fewer error at low exceedance of time for all the models, and the error increase with time percentages, indicate the differences between the measured and predicted values as the models were initially designed for temperate regions. Ghiani's model followed the ITU-R with close estimate to the measured rain attenuation. In addition, the performed error analyses showed similar judgment. Mello's model fails to adapt to the shorter link path, as it was empirically designed. The study highlights the need for a model that cover shorter path-length 5G links and designed especially for tropical regions. The results presented here could help in the design of a better rain attenuation prediction models for terrestrial links operating at higher frequencies in the tropics.

\section{ACKNOWLEDGMENT}

This work has been funded by Ministry of Education Malaysia and Universiti Teknologi Malaysia under "FRGS" Vot. No. RJ130000.7823.4F958, and Universiti Tun Hussein Onn Malaysia Tier 1 Grant Vot No. H160.

\section{REFERENCES}

[1] Rappaport TS, Sun S, Mayzus R, Zhao H, Azar Y, Wang K, et al. Millimeter wave mobile communications for 5G cellular: It will work! IEEE Access. 2013;1:335-49.

[2] Lam HY, Din J, Jong SL. Statistical and physical descriptions of raindrop size distributions in equatorial Malaysia from disdrometer observations. Adv Meteorol. 2015;2015:1-14.

[3] Moupfouma F, Tiffon J. Raindrop size distribution from microwave scattering measurements in equatorial and tropical climates. Electron Lett. 1982;18(23):1012-4.

[4] Lam HY, Luini L, Din J, Alhilali MJ, Jong SL, Cuervo F, et al. Impact of Rain Attenuation on 5G Millimeter Wave Communication Systems in Equatorial Malaysia Investigated Through Disdrometer Data. In: EuCAP. 2017. p. $1802-6$.

[5] Abdulrahman AY, Rahman TA, Rafiqul IM, Olufeagba BJ, Abdulrahman TA, Akanni J, et al. Investigation of the unified rain attenuation prediction method with data from tropical climates. IEEE Antennas Wirel Propag Lett. 2014;13(January 2014):1108-11.

[6] Capsoni C, Luini L, Paraboni A, Riva C, Martellucci A. A new prediction model of rain attenuation that separately accounts for stratiform and convective rain. IEEE Trans Antennas Propag. 2009;57(1):196-204.

[7] Kumar LS, Lee YH, Ong JT. Truncated gamma drop size distribution models for rain attenuation in Singapore. IEEE Trans Antennas Propag. 2010;58(4):1325-35.

[8] Awang M a, Din J. Comparison of the rain drop size distribution model in tropical region. RF Microw Conf 2004 RFM 2004 Proc. 2004;(D):20-2.

[9] Mello L da S, Pontes MS, Fagundes I, Almeida MPC, Andrade FJA. Modified rain attenuation prediction method considering the effect of wind direction. J Microwaves, Optoelectron Electromagn Appl. 2014;13(2):254-67.

[10] Ghiani R, Luini L, Fanti A. Investigation of the path reduction factor on terrestrial links for the development of a physically-based rain attenuation model. 2016 10th Eur Conf Antennas Propagation, EuCAP 2016. 2016;(1):2-3.

[11] Alhilali M, Din J, Schönhuber M, Lam HY. Estimation of Millimeter Wave Attenuation Due to Rain Using 2D Video Distrometer Data in Malaysia. Indones J Electr Eng Comput Sci. 2017;7(1):164-9.

[12] Lam HY, Luini L, Din J, Capsoni C, Panagopoulos AD. Application of the SC EXCELL model for rain attenuation prediction in tropical and equatorial regions. IEEE Asia-Pacific Conf Appl Electromagn APACE 2010 - Proc. 2010.

[13] ITU-R. Recommendation ITU-R P.530-17: Propagation data and prediction methods required for the design of terrestrial line-of-sight systems. Recomm ITU-R P530-17. 2017;

[14] Ghiani R, Luini L, Fanti A. A physically based rain attenuation model for terrestrial links. Radio Sci. 2017;52(8):972-80.

[15] ITU. P. 838-3 Specific attenuation model for rain for use in prediction methods. ITU-R Recommendations, P Series Fasicle. Geneva, Switzerland: ITU; 2005. p. 1-5.

[16] Amico MD, Jong SL, Riva C. Tipping Bucket Data Processing for Propagation Application. In: EuCAP. 2013. p. 256-60. 